\title{
Publisher Correction: Uncoupling protein 2 reprograms the tumor microenvironment to support the anti-tumor immune cycle
}

Wan-Chen Cheng, Yao-Chen Tsui, Simone Ragusa, Viktor H. Koelzer@D, Marco Mina, Fabien Franco, Heinz Läubli, Benjamin Tschumi, Daniel Speiser, Pedro Romero, Alfred Zippelius, Tatiana V. Petrova, Kirsten Mertz, Giovanni Ciriello (1) and Ping-Chih $\mathrm{Ho}$ (D)

Correction to: Nature Immunology https://doi.org/10.1038/s41590-018-0290-0 (2019), published online 21 January 2019.

In the version of this article initially published, the bars were not aligned with the data points or horizontal axis labels in Fig. $5 \mathrm{~d}$, and the labels along each horizontal axis of Fig. $5 j-1$ indicating the presence $(+)$ or absence $(-)$ of doxycycline (Dox) were incorrectly included with the labels below that axis. Also, the right vertical bar above Fig. $7 \mathrm{~b}$ linking ' $P=0.0001$ ' to the key was incorrect; the correct comparison is $\alpha \mathrm{PD}-1$ versus Dox $+\alpha \mathrm{PD}-1$. Similarly, the right vertical bar above Fig. 7e linking ' $P=0.0002$ ' to the key was incorrect; the correct comparison is $\alpha \mathrm{PD}-1$ versus Rosig $+\alpha \mathrm{PD}-1$. The errors have been corrected in the HTML and PDF versions of the article.
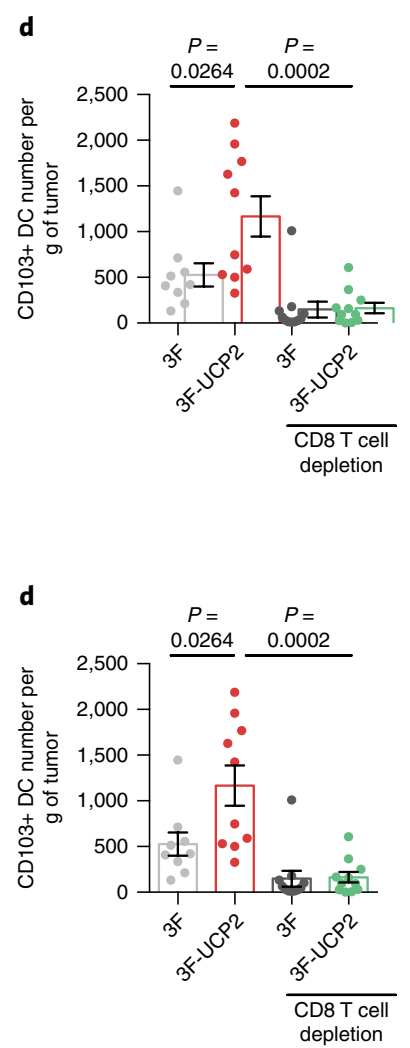
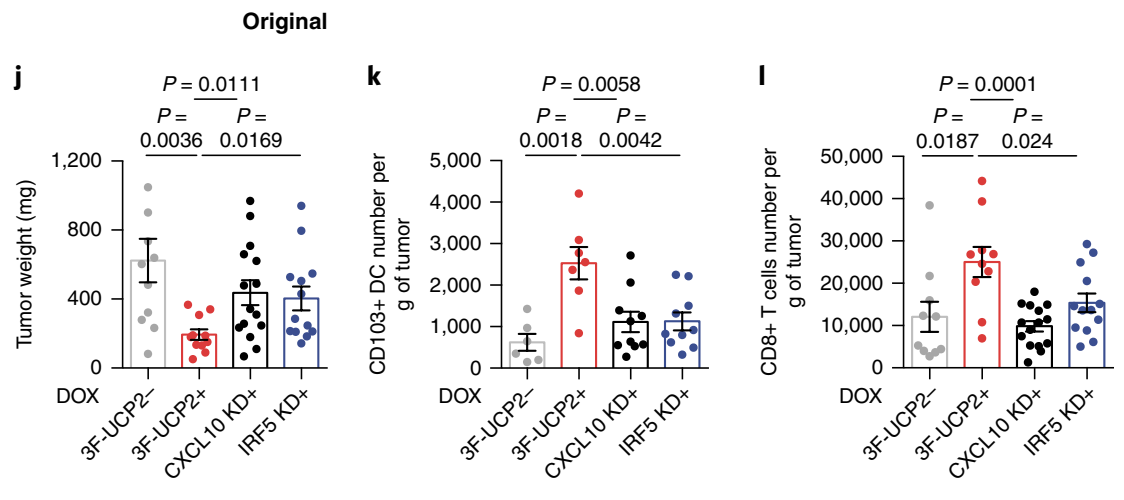

Corrected

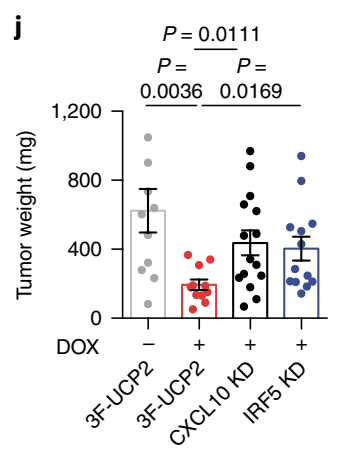

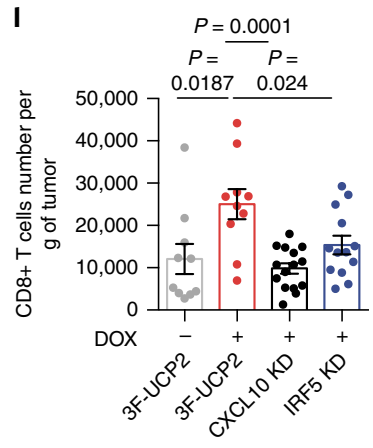

Fig. 5 | Original and Corrected. 

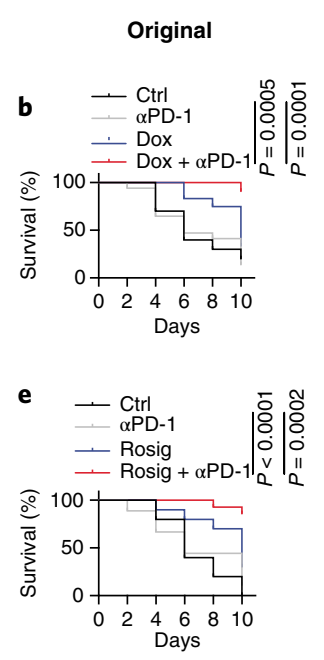

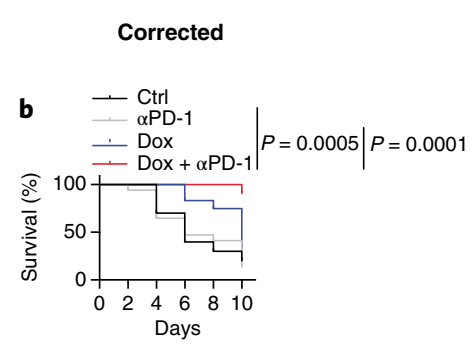

e

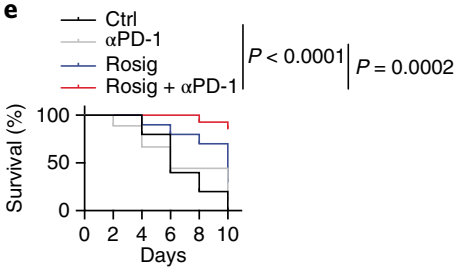

Fig. 7 | Original and Corrected.

Published online: 12 March 2019

https://doi.org/10.1038/s41590-019-0359-4

\title{
Publisher Correction: $\boldsymbol{\gamma} \boldsymbol{\delta}$ TCR ligands: the quest to solve a 500-million-year-old mystery
}

\author{
Benjamin E. Willcox $(\mathbb{D}$ and Carrie R. Willcox
}

Correction to: Nature Immunology https://doi.org/10.1038/s41590-018-0304-y (2019), published online 21 January 2019.

In the version of this article initially published, the affiliations were incorrect. The correct affiliations are as follows: " 1 Institute of Immunology and Immunotherapy, University of Birmingham, Birmingham, UK. ${ }^{2}$ Institute of Immunology and Immunotherapy, Cancer Immunology and Immunotherapy Centre, Cancer Research UK Birmingham Centre, University of Birmingham, Birmingham, UK.” The reference citation at the end of the first sentence of the second paragraph of the subsection 'A perspective on current methods of ligand identification' was incorrect; the correct citation is "...ligands ${ }^{20-40}$." There is an error (en dash) in the fourth paragraph of that section; the correct text is "...specific for CD1 and phycoerythrin.... There is an error ("proposed") in the fourth paragraph of the subsection 'An emerging adaptive perspective on antigenic $\gamma \delta$ TCR ligands'; the correct text is "...are suggested to recognize..." There is an error ("via") in the fourth paragraph of the subsection 'B7-like molecules as candidate $\gamma \delta$ TCR ligands'; the correct text is "...in a nonclonotypic fashion are striking.... Finally, reference citations throughout the legend to Fig. 1 are incorrect; the correct citations are as follows: MHC class I free heavy chain ${ }^{22}$; HLA-B5802 ${ }^{34}$; I-Ek (ref. ${ }^{30}$ ); MSH2 (MutShomolog 2) and HSP60 (heat-shock protein 60) ${ }^{24}$; ULBP4 (UL16-binding protein 4) ${ }^{27}$; $\mathrm{MICA}^{41}$; the herpes simplex virus glycoprotein $\mathrm{HSVgI}^{33}$; ATPase-apolipoprotein- $\mathrm{AI}^{39}$; and insulin B:9-23 peptide antigen ${ }^{40}$.

The errors have been corrected in the HTML and PDF versions of the article.

Published online: 28 February 2019

https://doi.org/10.1038/s41590-019-0358-5 